\title{
The estimation of dynamic contact angle of ultra-hydrophobic surfaces using inclined surface and impinging droplet methods
}

\author{
Darina Jasikova ${ }^{1,}$, Michal Kotek ${ }^{1}$ \\ ${ }^{1}$ The Institute for Nanomaterials, Advanced Technologies and Innovation, Technical University of Liberec, Studentska \\ 1402/2, Liberec 1, Czech Republic
}

\begin{abstract}
The development of industrial technology also brings with optimized surface quality, particularly where there is contact with food. Application ultra-hydrophobic surface significantly reduces the growth of bacteria and facilitates cleaning processes. Testing and evaluation of surface quality are used two methods: impinging droplet and inclined surface method optimized with high speed shadowgraphy, which give information about dynamic contact angle. This article presents the results of research into new methods of measuring ultra-hydrophobic patented technology.
\end{abstract}

\section{Introduction}

So far the investigation of hydrophobic surfaces in fluid mechanics was solved on the macroscopic level. The problem of hydrophobic surfaces is very complex and can be crucial to fluid mechanics, especially the problem of adhesion of liquid on a solid surface that is set as boundary condition in the most of mathematical models.

The interdisciplinary project GA13-20031S deals with the research of hydrophobic surfaces. Based on feedback, teams cooperate and produce the coatings on the principle of using nano-plasma technology [1]. The aim is to gain basic knowledge about the movement of fluid in the contact layer between the liquid and the hydrophobic surface [2].This knowledge is used to reduce hydraulic losses and the change in concentration of gases in liquids. Surface binding properties are studied in terms of surface energy, the adhesion coefficient. To the experimental study and analysed data are set in the mathematical model with new boundary conditions, using the adhesive coefficient, determining adhesion strength in the surface layer of hydrophobic surface to prove and validate the physical expressions [3]. This creates the prerequisites for the establishment of wall functions necessary for the formulation of the updated model of turbulence. Due to the traction fluid on the wall with hydrophobic properties, it changes the boundary layer and its stability. In addition, there will be changes in pressure, which forces the study of problems of cavitation. From the measurements it is possible to estimate the value of the contact angle of approximately $160^{\circ}$ this corresponds with characteristics of super hydrophobic surface.

In 1823, set the Navier the basic relation for the liquids. Approximately 200 years ago, Thomas Young suggested a relationship for the contact angle of the liquid [4]. In 1937, Bangham, D. H., Razouk, R.I. pointed out the importance of negligence gas adsorption on the surface of the solid phase during the derivation of Young's equation [5].In the following years have been done a lot of research on the measurement of fluid slip; both from the theoretical, and experimental point of view. However, the results were not very successful because there were not known the surfaces with sufficient degree of hydrophobic [6].

The measurements were performed with a small contact angle, so the fluid slip was negligible and measurable with a big mistake. Also, experimental methods were not sufficiently accurate [7]. These measurements clearly showed "nonslip" boundary condition. Additional experiments revealed that if the interactions between the liquid and the solid surface will be weaker, there is an increase of the slip; the hydrophobic surfaces have a length slip beneficial effect [8].

During the experimental examining of the surface roughness influence on the slip length, the authors diverge in their conclusions. Some experiments have shown that the surface roughness decreases the length of the slide; other experiments showed the opposite results. This phenomenon can be explained such that at full wetting of rough surface leads to reduction of the slip, while the partial wetting of the surface roughness creates favourable conditions for the formation of gas or vapour, resulting the increase of the slip length [9].

The hydrophobic surfaces are characterized with following properties: hydrodynamic drag that is an important issue in many fluid systems. The examination

\footnotetext{
${ }^{\mathrm{a}}$ Corresponding author: darina.jasikova@tul.cz
} 
of this property can be used goniometer system [10]. The typical property of the surface is the contact angle.

The contact angle measurements involve the sessile drop or pendant drop technique that is widely used for investigation of the surface characteristics of various materials. Measuring this property we can also determine the degree of water repellence [11].Another point of view on the evaluation of the hydrophobic surfaces offers the experiments based on the online study of drop impact.

This kind of studies involving the drop impinging and the movement of the defined droplet on the inclined surface. Impacts of individual water droplets on hydrophobic surfaces at slow velocities allow identification of three distinct regimes of droplet behaviour: rebound, pinning and fragmentation. This set of inner conditions enable us state the kinetic energy that is converted to deformational potential energy, due the surface tension for liquids and elasticity for solids, and appears as the translation motion and the internal drop motion (waves, oscillations and dissipation).

\section{Sample preparations}

In this work are published results taken on the surfaces produced by the plasma deposition technologies. The plasma deposition can be provided by many various subtechnologies, such as CVD, PVD, PECVD, spraying, spinning, etc. Each named technology includes a wide range of specific technologies, which differ from each other as technological equipment and working conditions, as well as the achieved results, i.e. the types and composition of the various deposits and their properties, structure, and chemical composition. Only some of them exhibit nanostructured character.

The one way of making nanostructured thin films especially with varying degrees of hydrophobicity of the above plasma jet systems. These nanostructures are suitable to modify any surface and make its basic value. Another interesting form of nanostructures particularly in terms of supporting or reinforcing permeable structures are nanowire, which today can be prepared on an industrial scale for many materials. Another example of well-known application techniques nanostructured layers are methods of creating a very fine aerosol that is applied to the surface of the material. Practically, only two aerosol generation technologies of hitherto commonly used technologies allow you to generate sufficiently fine and homogeneous aerosol with a narrow size distribution of aerosol particles, which is suitable for creating defined nanostructures, nanostructured layers or nano composite materials.

The first generation of appropriate technology is called aerosol electrostatic spraying in which either uses electrospray nozzle or aerosol generated from a "free“" level using a modified electro spinning technology [12].

Using the plasma jet at atmospheric pressure for a modification of macromolecular materials and surfaces is disclosed in EP 07466017.6-1226 (Klima, M. et al. 2007). This application provides a method including coating plasma structured macromolecular substances on substrates of macromolecular substances. The plasma jet or multi-jet system that generates plasma at atmospheric pressure is based on principle of the hollow cathode running with high frequency $(13.56 \mathrm{MHz})$ - see Patent GB 286310, U.S. 6,525,481 (2003), EP 1077021 (2005).

The basis of jets currently uses dielectric capillaries of silica or alumina, which working gas argon that flows through the electrode. Dielectric capillary locked around annular electrode or multi-electrode system. The discharge is ignited pre ionized of environment in dielectric capillary nozzle. The resulting plasma springs from the cavity and the mouth of the plasma jet to the external environment in which it operates on the surface. Grouping of jets in the linear profile or matrix allows working on larger areas. In the present study the new linear types of plasma jets allowing greater homogeneity of surface treatment and easier combination for aerosol type precursors.

In this paper are published results measured with following samples. Sample No.1 - ultra hydrophobic layer obtained by direct deposition of HMDSO precursor of argon plasma generated by plasma jets (multi jet-row system 20 dielectric capillaries on the width $10 \mathrm{~cm}$ ). Sample No.2and 3 were prepared by the same procedure, i.e., first by the plasma, and the precursor was formed HMDSO ultra hydrophobic layers, subsequently it was applied to two different types according nanopolymer sample (to improve stability and mechanical properties). For sample No.2 was used nanopolymer based on $\mathrm{SiO} 2$ in ethanol solution (classical Nano-glass) for the sample No. 3 was chosen based nanopolymer Fluor hydrocarbons in aqueous solution (rubbed into the structure ultrahydrophobic layer). Samples No.4 and 5 were prepared by the same procedure, i.e., first by the plasma, and the precursor was formed HMDSO ultra hydrophobic layers will, subsequently it was applied to two different types according nanopolymer sample (to improve stability and mechanical properties), the sample consists was also briefly a modified plasma precursors HMDSO. For sample No. 4 was used nanopolymer based on $\mathrm{SiO} 2$ in ethanol solution (classical Nano-glass) for the sample No. 5 was chosen nanopolymer based Fluor hydrocarbons in aqueous solution (rubbed into the structure ultrahydrophobic layer). Sample No.6 was formed by first using plasma and precursor HMDSO was created ultrahydrophobic layers will, subsequently it was applied to a thin layer of plasma precursor trimethyl boric acid (for reinforcement structure).

\section{Experimental setup}

For the investigation of the manufactured samples we used the optical method. The basic set up of the shadowgraph method was completed and upgraded with high speed camera SpeedSense - DantecDynamics and software PCC2.1.

The data obtained from the measurement were mathematically processed and analysed. The continuous light source of $1.5 \mathrm{kWatt}$ was focused to the path of the drop and placed oppositely to the camera. Using this setup with very small exposure time and the aperture number 4 we obtain quality image with high contrast. 
Images were received and digitized by a CCD high speed camera. The whole system, including storage of images, was controlled by a personal computer. The sample rate was set $5000 \mathrm{fps}$; the exposure time of the CCD-camera was $1.02 \mu$ s with image resolution $(1280 \times 800)$ pixels. During the measurement we used the pre trigger of $1 \mathrm{sec}$ time and the measurement was synchronized by the photocell.

For the examination of the sample we used the impinging droplet method and the method of inclined surface.

The impinging drop method give us information about wettability of the substrate that can be also described with the contact angle and the second condition that can influence the droplet behaviour is the surface average roughness. Using this method we can study the process of droplet kinetic energy dissipation by viscous effects and surface energy that is demonstrated by dynamic behaviour.

The inclined wall method utilizes the conversion of translational kinetic energy into deformational potential energy. Here we used setup with the sample inclined under $20^{\circ}$. In both experimental setups the droplet was generated with the blunt needle nozzle of diameter 0.3 $\mathrm{mm}$. For the inclined surface method the needle tip was placed $4 \mathrm{~mm}$ above the surface to avoid the impinging and oscillating factor of the droplet movement. In the impinging droplet method, the needle tip was fit $15 \mathrm{~mm}$ above the surface.

In both cases as the medium liquid was used distilled water.

\section{Results and discussion}

Each sample mentioned above was tested with the impinging droplet method as well as with water drop motion on the inclined surface.

a)

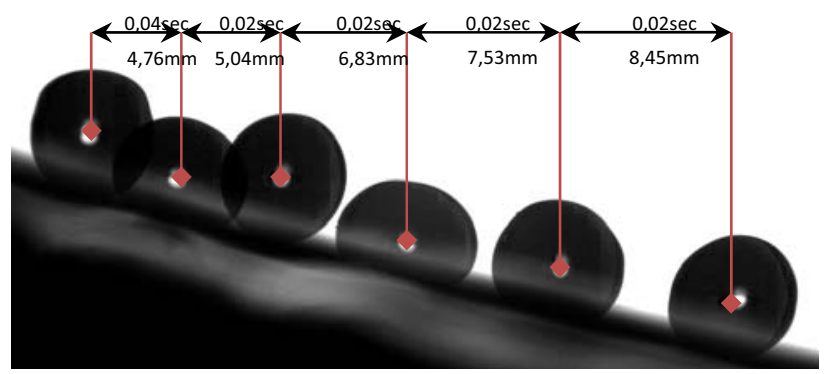

b)

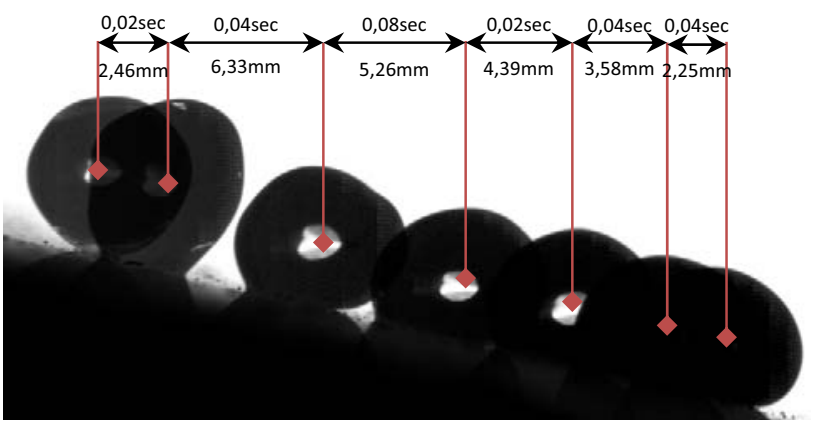

c)

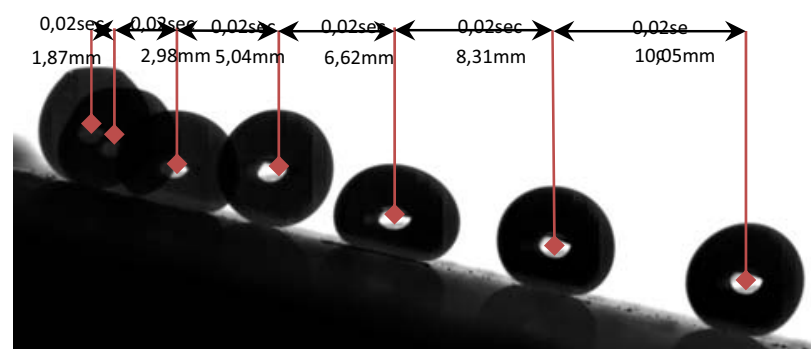

Figure 1. The example of the shadowgraphy measurement of the droplet motion on the inclined surface - sample No. a) 3, b) 4 and c) 6

Using the inclined surface method we achieved the information about the basic characters of the droplet movement on the investigated surface. Even from this basic primitive observation we recognized the main difference between the samples. This measurement also uncovered instabilities and no uniformities of the provided samples. During the measurement we followed the path of the single one droplet and afterwards the sample was moved to the original path with micrometric traverser. This setup enabled scanning the whole sample condition so we got the complex information about the sample. The resulting chart of velocities is a statistic of the 15 measuremnts, where each measurement represents one droplet path.

\section{Droplet velocity}

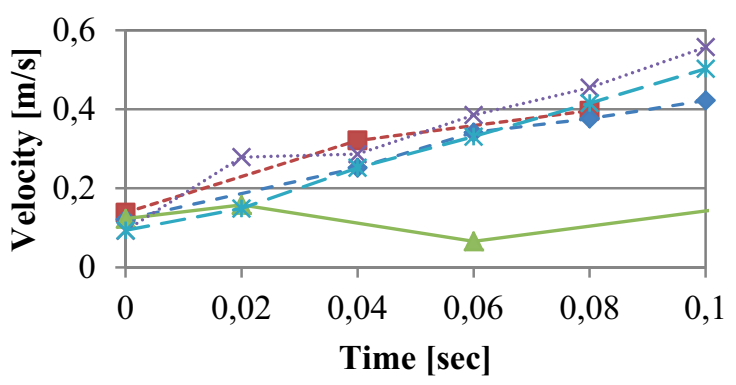

\begin{tabular}{|c|c|}
\hline ---1-- Sample 1 & $-\neg-$ Sample 3 \\
\hline$\longrightarrow$ Sample 4 & … × .... Sample 5 \\
\hline
\end{tabular}

Figure 2. The velocity of the droplet movement on inclined surface under $20^{\circ}$.

The second part of sample testing involved the impinging droplet method. The images obtained by high speed camera with sampling rate 5000 were analysed in image processing software and were calculated both contact angle between the liquid and solid phase. The charts of result containing the time part, when the droplet is in the contact with substrate. The default point of measurement was set the first contact of droplet with surface with $1 \mathrm{px}$ accuracy. The contact angle processing was finished, when the droplet left the surface. As the reference sample was determined the pure glass. 
From the computing chart is well seen the respond of contact angle on the oscillation of the droplet, where predominates the elastic part of the kinetic energy.

Expected characteristic behaviour of the impinging droplet can be categorized in two types: corona splashing and prompt splashing. The observed behaviour of the water droplets has no significant features that can be easily classify into this two categories. The behaviour of the droplet remains mostly the liquid with higher viscosity. The splashing factor may be observed in different set up, means with the needle tip placed higher than $15 \mathrm{~mm}$ above the substrate. Analysing the captured images we were interested in the time track $0.06 \mathrm{sec}$, because here predominates the impact of the surface on the droplet behaviour. This impact also reflects the contact angle of the liquid.

Pure glass
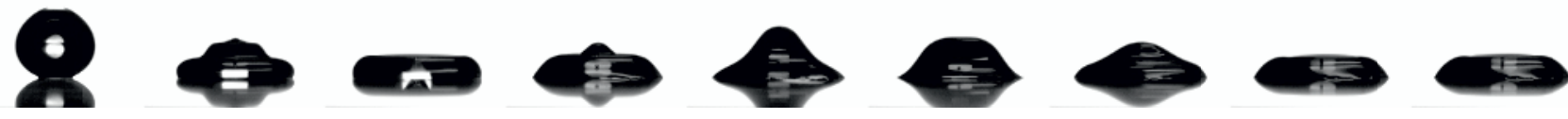

Sample 2

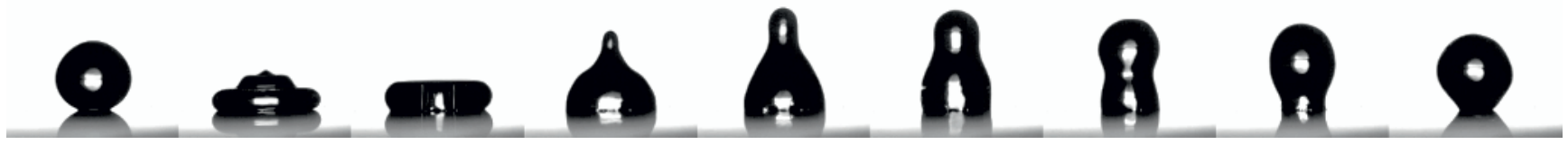

Sample 3

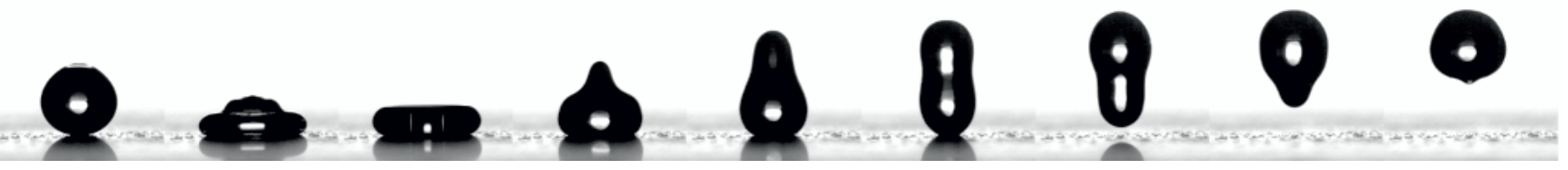

Sample 4

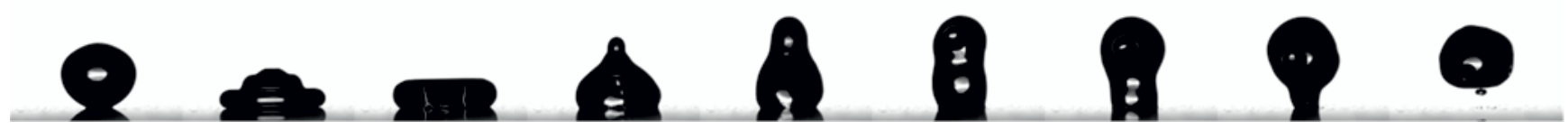

Sample 5

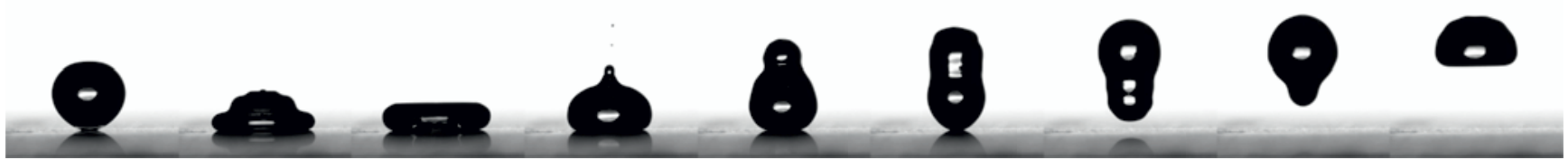

Sample 6

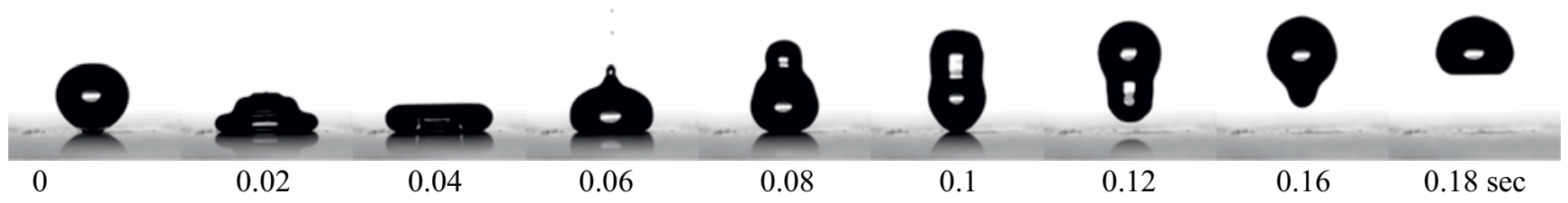

Figure 3. The complex image of the water drop behaviour on the investigated surfaces using the impinging drop method. 


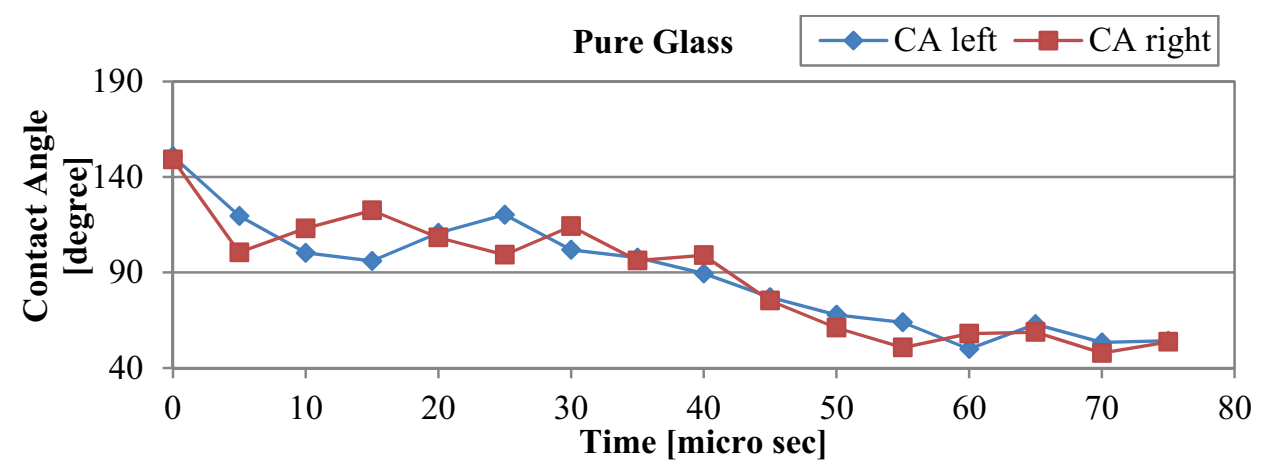

Figure 4. The graph of the dynamic contact angle - left (CA left) and right (CA right) for the interaction between water and pure glass surface.

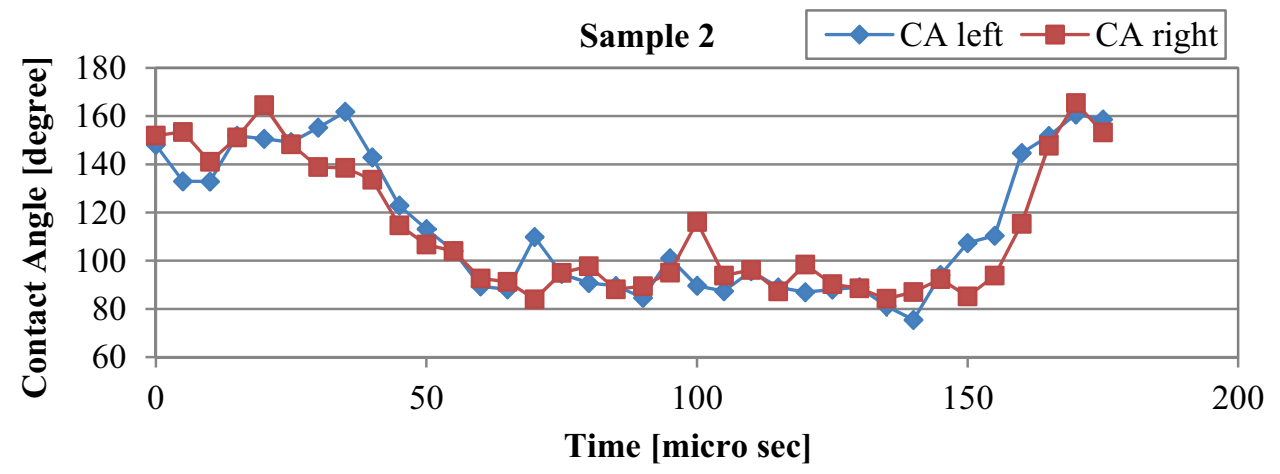

Figure 5. The graph of the dynamic contact angle - left (CA left) and right (CA right) for the interaction between water and sample no. 2 that was prepared by plasma treated precursor nanopolymer based on $\mathrm{SiO}_{2}$ in ethanol solution (classical Nano-glass).

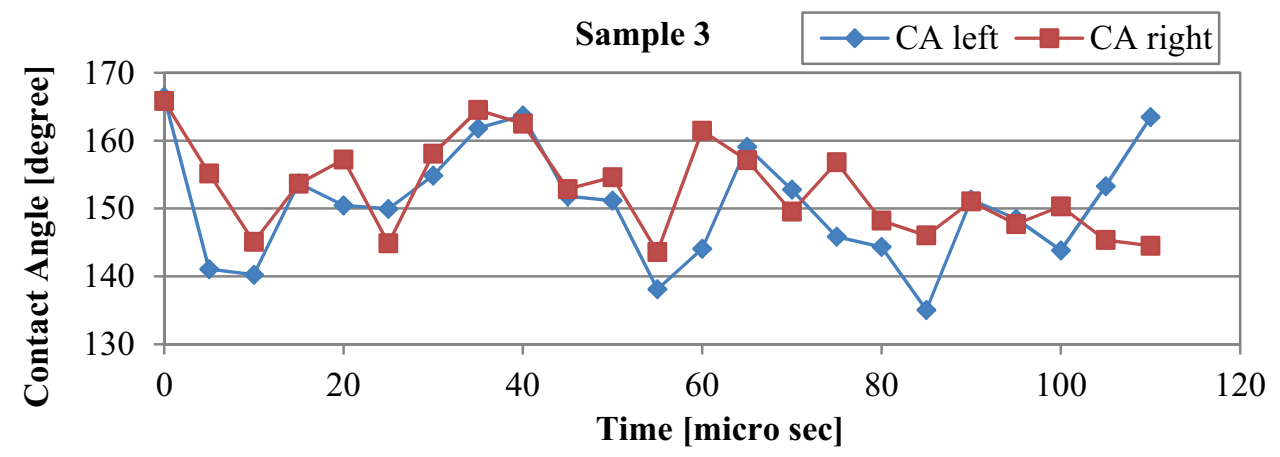

Figure 6. The graph of the dynamic contact angle - left (CA left) and right (CA right) for the interaction between water and sample no. 3 that was prepared by plasma treated precursor nanopolymer Fluor hydrocarbons in aqueous solution.

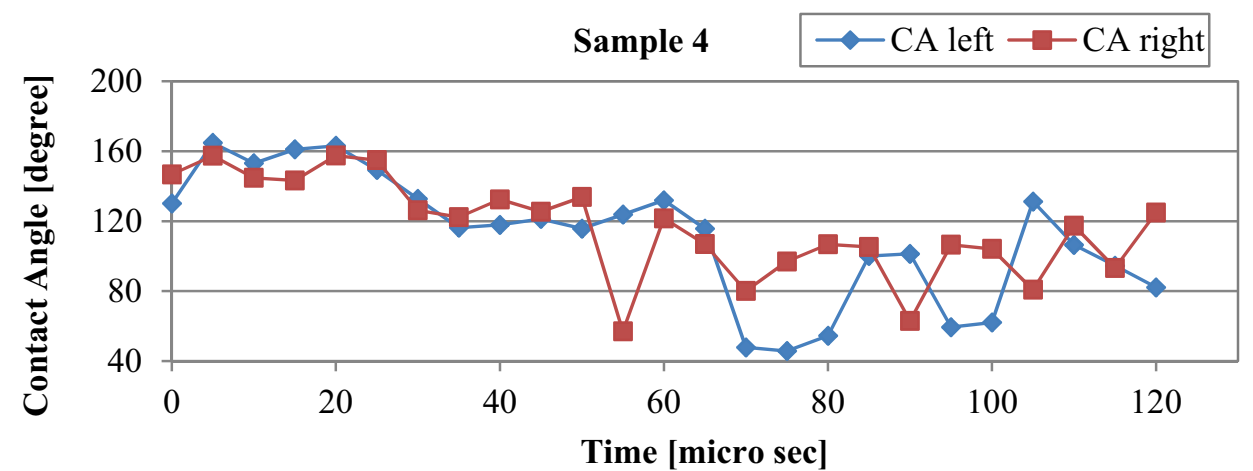

Figure 7. The graph of the dynamic contact angle - left (CA left) and right (CA right) for the interaction between water and sample no. 4 that was prepared by plasma treated precursor nanopolymer based on $\mathrm{SiO}_{2}$ in ethanol solution on rubbed substrate.

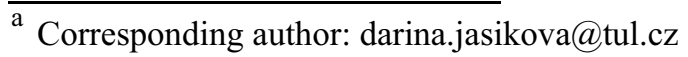


The second important time track is the $0.12 \mathrm{sec}$, when the droplet leaves the substrate with characteristic profile and jumps in the certain height. This height of the first jump can be further analysed, because it seems to be another important characteristic of the super hydrophobic surface. This behaviour follows the wall friction law and slips effects and should be involved in the future studies as well as the physical analyses of the process.

The measurements of contact angles are shown in the graphs below. The selected results refer the different behaviour of the liquid phase. The first chart named "Pure Glass" follows the characteristic development of the sessile drop on the solid substrate with corresponding contact angles and interfacial behaviour that stabilize after 60 microsecunds on $58^{\circ}$.

The samples No. 3, 5 and 6 show the same characteristics in the meaning that the contact angle profile is stable and varying about certain value that is significant for the sample. These samples demonstrate the typical statement of super hydrophobicity with the contact angles of value round $160^{\circ}$, and the low edge of hydrophobic value of contact angle was set to be $130^{\circ}$.

The sample No. 2 shows the characteristic of the hydrophobic surface, but without the antipearl effect that is very important in industrial applications. The droplet stays close the surface with great contact area.

Another interesting behaviour we can observe with sample No. 4. This behaviour lies between the sample No. 2 and antipearl effect. The liquid phase reacts on the contact with solid substrate with developing thin stem. This stem is finally cut, but stays significantly present on the profile of the impinged droplet.

\section{Conclusions}

This article brought information about novel patented method of super hydrophobic surface production. This method is suitable for industrial application requiring self-cleaning process.

We have described two methods that are useful for studies and visualization basic properties of these surfaces. They are both based on the optical measurement and further image processing.

The initial analyses uncovered the weakness of both method but also pointed the useful information that can be used in mathematical simulation for statement of boundary condition.

\section{Acknowledgment}

The author gratefully thank to the support of the Czech Science Foundation GA13-20031S, Centre for nanomaterial's, advanced technologies and innovation CZ.1.05/2.1.00/01.0005 and CZ.1.07/2.3.00/30.0024.

\section{References}

1. M. Klíma,et al, US 6,525,481, EP 1077021 (2005)

2. M. Klíma, et al., EP 07466017, (2007)

3. F. Pochyly, et al., International Journal of Fluid Machinery and Systems, 3(4), 386 - 394 (2010)

4. C. L. M. H. Navier, Mémoeres de l'Académie Royale des Liquids, VI, 389-440, (1823)

5. D. H. Bangham, R. I. Razouk, Trans. Faraday Soc. 33, (1937)

6. N. V. Priezjev, S. M. Troian, Phys. Rev. Lett. 92, (2004)

7. D. Y. C. Chan, R.G., J. Horn, Chem. Phys. 83 (1985)

8. R. Truesdell, et al., Phys. Rev. Lett. 97 (2006)

9. C. Cottin-Bizonne, et al., Nat. Mater. 2 (2003)

10. E. Aljallis, et al., Physics of fluids 25, (2013)

11. C. A. E. Hamletta, et al, European Journal of Soil Science, 64, 324-333 (2013)

12. D. Jasikova, V. Kopecky, Experimental fluid mechanics, 98-108 (2009)

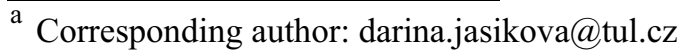

